\title{
BEHAVIOUR AND FATE OF LYMPHOCYTES
}

$\mathrm{L}^{\mathrm{Y}}$

YMPHOCY'TES make up a substantial proportion of the leucocytes in mammalian blood. Lymphocytes are not, however, confined to the blood: they are present diffusely throughout the submucosa of the intestine and in the bone marrow; they are the predominant cell-type in the normal lymph node, in the Peyer's patches of the intestine and in the lymphoid tissue in the lung and in tho spleen. In pathological material they are particularly associated with areas of chronic inflammation and with eertain tumours. Despite this widespread distribution in both normal and abnormal tissues, very little is known about the lifo-history of the lymphocyte and virtually nothing about its function. The problem is made more striking by the apparently high rate of replacement of the blood lymphocytes; in the rat, for example, the output of lymphocytes from the main lymphatic vessels is sufficient to replace all the lymphocytes in the blood at least eleven times daily. So far as the fundamental properties of lymphocytes are concerned, there would only be agreement that they are motile, non-phagocytic cells which do not respond chemotactically to any stimulus yet tried.

Lymphocytes are produced in the lymph nodes. These are complex structures containing several apparently distinct cell-types : the phagocytic cells lining the lymph channels enable the lymph nodes to filter foreign material from the fluid percolating through them; there is very good evidence that plasma cells are responsible for the production of circulating antibodies; but there is, as yet, no function of lymphoid tissue that can be definitely attributed to its content of lymphocytes.

'The problem of the behaviour and fate of lymphocytes was discussed at a Ciba Foundation Study Group held in London on July 23 under the chairmanship of Prof. P. B. Medawar.

The appearance of living human lymphocytes in vitro was well illustrated in a film by R. J. F. Pulvertaft (London). The cells were examined in a narrow layor of culture fluid enclosed between a glass coverslip and an agar surface. Time-lapse cinematography showed that lymphocytes often moved towards, and adhered to, malignant cells, to megakaryocytes and to cells of all kinds in mitosis. Having adhered to these cells, the lymphocytes often moved continuously around their periphery, a phenomenon for which the term 'emperipolesis' has been coined. 'The discussion of this paper centred mainly on whether or not emperipolesis might be an artefact due to the special conditions existing in the agar-cover-slip chamber. G. M. Watson (Harwell) showed that Iymphocytes in such preparations will gyrate around glass beads; he suggested that when large cells or glass beads are compressed into the agar by the cover-slip a circular trough is formed around them in which lymphocytes can become trapped. The lymphocytes cannot escape from this trough bocause, unlike other leucocytes, they will not adhere to glass surfaces; they therefore continue to circle around in it.

The part played by the thymus in the production of lymphocytes was discussed by E. Andreasen
(Copenhagen). The high turnover of deoxyribonucleic acid phosphorus and the high mitotic activity in the thymus suggested that it was an important source of the lymphocytes in the lymph and the blood. However, the measurement of the concentration of lymphocytes in the blood and the lymph of thymectomized rats led to the conclusion that very few thymus-lymphocytes leave the organ, and that the production of now cells in it is more or less balanced by a continuous destruction of lymphocytes by the reticulum cells. It was pointed out that the concurrent now formation and disintegration of lymphocytes appears to be a widespread phenomenon in lymphoid tissue. O. A. Trowell (Harwell) described the occurrence of such a process in rat lymph nodes cultured in vitro. The histological appearance of these cultures suggested that the reticulum cells were ablo to phagocytose the remains of dead lymphocytes and then to differentiate into large lymphocytes. The transformation of small lymphocytes into macrophages was also thought to occur, and it was suggested that the small lymphocytes might constitute, in the animal, a reserve of potential macrophages. The discussion showed that tho possibility of lymphocytes transforming into other cell-types remains a highly controversial topic.

The transplantation of myeloid and lymphoid tissue was discussed by J. F. Loutit (Harwell) and N. A. Mitchison (Edinburgh). Following a lethal dose $\left(L D_{98}\right)$ of X-rays, mice can be induced to recover by an intravenous injection of myeloid cells. Recovery is due to the recolonization of the damaged myeloid and lymphoid tissue by the cells of the donor. When these cells are derived from the same inbred strain, the recolonized animals live nearly as long as normal mice. But when the transplanted cells come from another strain of mice, or from the rat, many animals die 1-3 months later. It was argued that this secondary death was the result of an immunological reaction against the antigens of the host by the transplanted cells. In non-irradiated hosts, transplanted lymphoid cells also become localized in lymphoid tissue. They are capable of immunological activity, for they continue to produce antibody when taken from donors which have been actively immunized. Unless taken from the same inbred strain as their host, the transplanted cells are soon eliminated by the homograft reaction.

L. D. Hamilton (New York) and J. Ottesen (Copenhagen) described the incorporation of radioactive isotopes into the nucleic acids of lymphocytes in the blood. Hamilton administered ${ }^{14} \mathrm{C}$-adenine to patients with chronic lymphatic leukæmia and isolated the purines from the deoxyribonucleic acid and the ribonucleie acid of the circulating leukiemic lymphocytos. The radioactivity of the purines in both the ribonucleic acid and the deoxyribonucleic acid declined in two phases, rapidly at first, and then slowly, with a very prolonged retention of radioactivity. An analysis of these curves suggested that there were two populations of lymphocytes, one with an average life-span of eighty-five days, and the other with a half-life of about three hundred days. A great deal of interest contred around Hamilton's suggestion that 
the prolonged retention of radioactivity in the lymphocytes might not indicate cells with a very long life-span but might be due to a continuous re-utilization of large fragments of nucleic acid or nucleoprotein during the formation of new lymphocytes. The biochemical evidence did not enable a choice to be made between these alternatives, but Trowell suggested that the presence of cell debris in the reticulum cells in lymph nodes could provide cytological support for the idea of re-utilization.

Ottesen described the incorporation of phosphorus32 into the deoxyribonucleic acid of lymphocytes in the blood of normal human subjects. An analysis of the specific activity curves again suggested the existence of two populations of cells but with shorter life-spans than those calculated by Hamilton for leukæmic lymphocytes : about 20 per cent had a mean age of $2-3$ days and 80 per cent a mean age of 100-200 days. There was evidence, both in man and in animals, that lymphocytes may remain in lymph nodes for a considerable time after their formation. Thus, autoradiographs of mouse lymph nodes showed that some lymphocytes labelled with carbon-14 were present in the nodes more than two months after the administration of the isotope.

These experiments with radioactive labels suggested that the life-span of at least some lymphocytes may be considerably longer than was at one time supposed. There was, however, some prejudice against the idea that lymphocytes can live for as long as a year, and, in discussion, this appeared to be a reason for inclining towards the hypothesis of re-utilization.

The problem of the rapid turnover of the lymphocytes in the blood was discussed by J. L. Gowans (Oxford). An apparatus was described for the continuous intravenous infusion of lymph and lympho. cytes into unanæsthetized rats with thoracic duct fistulæ. It was shown that there is a direct relationship between the number of lymphocytes entering the blood and the number emerging from the thoracic duct. Thus, the fall in the cell output that occurred on the prolonged drainage of lymph from the thoracic duct could be prevented by the intravenous replacement of all the lymph and cells collected from the fistula. Similarly, after prolonged drainage of lymph, the low output of cells from the thoracie duct could be restored to normal by the transfusion of lymphocytes from other rats of the same inbred strain. A vigorous transfusion of lymphocytes almost doubled the normal cell output of a freshly cannulated rat. The number of lymphocytes transfused could be roughly accounted for by the extra lymphocytes appearing in the thoracic duct lymph of the recipient rat. These effects were most probably mediated by living lymphocytes since the administration of cellfree lymph or of lymphocytes killed by a variety of means had no effect on the cell output. It was felt that the results could not be accounted for by a burst of new cell formation initiated by the trans. fused cells ; they strongly suggested that the apparently high turnover of the lymphocytes in the blood of the intact animal is due to a continuous recirculation of lymphocytes from blood to lymph to blood. This idea was further strengthened by an experiment in which the transfusion of radioactively labelled lymphocytes resulted in their rapid appearance in the thoracic duct lymph.

J. M. Yoffey (Bristol) said that lymphocytes probably recirculated on a small scale via the afferent (peripheral) lymphaties, but that there were a number of difficulties in accepting the hypothesis that recirculation occurred on the scale envisaged by Gowans. Detailed histological observations on the regenerating bono marrow of irradiated guinea pigs tends to suggest that lymphocytes provide a reservoir of stem cells which contribute to the regeneration of marrow.

In the experiments described at the meeting and in discussion it was again mado plain that there is, as yet, no real clue to the physiological function of the lymphocyte. Prof. Medawar, in his concluding remarks, expressed the appreciation of those present for the hospitality given by the Ciba Foundation.

\section{ELECTROLYTE PHYSIOLOGY}

$\mathrm{A}^{1}$ 'T the British Association meeting in Dublin, the session held by Section I. (Physiology and Biochemistry) on September 9, on "Electrolyte Physiology", opened with a paper by Prof. E. J. Conway (University College, Dublin) on "Energy and Form in the Living Cell". In a paper on so wide a subject, the treatment was necessarily limited to certain aspects, but it includes some basic considerations of ionic permeability of the animal-cell membrane.

The energy requirements of the living cell could be listed under the following general headings : $(a)$ maintenance of size and composition ; (b) special functions such as contraction, conduction and secretion; and (c) cell growth and division. The treatment was limited to the first heading, the fully developed cell being considered in general, apart from special functions.

Energy required for maintenance of size with respect to water-content. It is reasonable to suppose that the primitive cell was surrounded by a membrane, neces- sary to retain substances required for its activities. This membrane is considered to be freely distensible, the comparatively rigid plant-cell membrane developing later as an adaptation to sites with variable mixing of sea and fresh water.

As the membrane may be assumed to exist primarily for conserving essential cell substances, it should have some limiting permeability. If it were quite impermeable to solutes, necossary material would be excluded, without some special expenditure of energy. Passing from such permeability to one in which sodium and potassium ions as well as small anions could enter with equal facility per unit concentration, then, from considerations of osmotic, electrical ant Donnan equilibria, the following equation can be written ${ }^{1}$ for the equilibrium condition

$$
\eta=C-\left(\varepsilon^{2}+4 \Sigma b d\right)^{1 / 2}
$$

Here $\eta$ represents moles of non-diffusible material within the cell (using the term non-diffusiblo with reference to the mernbrane), $\varepsilon$ is the arithmetical sum 